\title{
Estimation of Multiple Sources Using Spatio-Temporal Data on a Three-Dimensional Measurement of MEG
}

\author{
Koichiro Kobayashi and Yoshinori Uchikawa, Member, IEEE
}

\begin{abstract}
This paper describes a computer simulation study for estimating the multiple sources in the brain based on a three-dimensional measurement of magnetoencephalogram (3-D MEG). We propose a new source analysis method using spatio-temporal data of 3-D MEG and singular value decomposition analysis. The number of estimation sources is decided by the number of dominant singular values of spatio-temporal 3-D MEG data. The source localization is done as pattern matching by signal subspace of singular vectors and projection of an estimating source. In order to assess this algorithm, inverse calculation to the MEG model consisting of three sources located in the brain was performed. The results showed that three sources were clearly discriminated, and that the estimation error was less than $1 \mathrm{~mm}$ with SNR $=5$. The proposed method is useful for estimating multiple sources overlapping in time.
\end{abstract}

Index Terms-3-D MEG, multiple sources estimation, singular value decomposition analysis, spatio-temporal data.

\section{INTRODUCTION}

M EG MEASUREMENT of the magnetic field perpendicular to the scalp is widely used. We have earlier developed a three-dimensional vector measurement system that can detect magnetic field component perpendicular $(\mathrm{Br})$ and tangential $(\mathrm{B} \theta, \mathrm{B} \phi)$ to the scalp simultaneously [1]. A lot of algorithms for the multiple sources estimation have been proposed; e.g., the early [2]-[4]. There are problems in estimating multiple sources overlapping in time when many distinct areas of cortex are active as discussed e.g., in [5]-[7]. We propose a new estimation algorithm for multiple sources using spatio-temporal data of 3-D MEG and singular value decomposition analysis. This algorithm uses tangential component to decide the initial parameters for source analysis. The tangential component provides information about the source location by visual inspection [8]. The computer simulation study was done on a model comprising three active sources overlapping in time. The location errors were examined.

Manuscript received February 7, 2001.

This study has been supported by a grant from the Japan Ministry of Education, Science and Culture Grant-in-Aid for High-technology Research Center and Scientific Research, the Advance Technology Research and the Center for Research of Tokyo Denki University.

K. Kobayashi is with the Applied Superconductivity Research Laboratory, Tokyo Denki University, Chiba, Japan (e-mail: kobaya@asrl.dendai.ac.jp).

Y. Uchikawa is with the College of Science and Engineering, Tokyo Denki University, Saitama, Japan (e-mail: uchikawa@f.dendai.ac.jp).

Publisher Item Identifier S 0018-9464(01)07117-5.
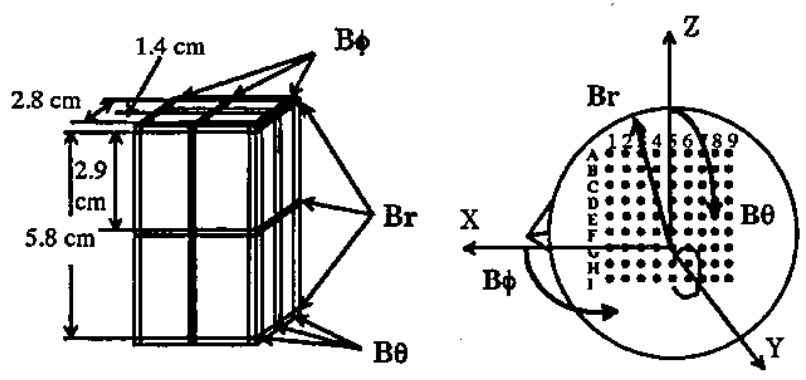

Fig. 1. A 3-D second-order gradiometer and coordinate system and the measuring grid comprised 81 points on a spherical model of a $10 \mathrm{~cm}$ radius.

\section{METHOD}

In the simulation study the human head is modeled as a spherical homogeneous conductor with a radius of $10 \mathrm{~cm}$. The curvilinear measuring grid consists of 81 points with a $2 \mathrm{~cm}$ separation between the nearest neighbors. In calculating the magnetic field detected by a pick-up coil on the scalp, the second-order gradiometer configuration developed by authors is taken into account [8].

The matrix $\mathbf{B}$ represents the spatio-temporal magnetic field data. Each column vector shows spatial information of magnetic field at a given instant of time and the row vectors show temporal evolvement of magnetic field at a given measurement point

$$
\mathbf{B}=\left[\begin{array}{cccc}
b_{11} & b_{12} & \cdots & b_{1 K} \\
b_{21} & b_{22} & \cdots & b_{2 K} \\
\vdots & \vdots & \ddots & \vdots \\
b_{J 1} & b_{J 2} & \cdots & b_{J K}
\end{array}\right]
$$

Where $J$ : number of measuring points, $K$ : number of samples. The singular value analysis of matrix $B$ is given by

$$
\begin{aligned}
& \mathbf{B}=\mathbf{U} \Omega \mathbf{V}^{T} \\
& \boldsymbol{\Omega}=\operatorname{diag}\left(\sqrt{\lambda_{1}}, \sqrt{\lambda_{2}}, \ldots, \sqrt{\lambda_{I}}\right) .
\end{aligned}
$$

Each column $\mathrm{u}$ of $\mathrm{U}$ is the eigenvector that corresponds to the nonzero eigenvalue of $\mathrm{BB}^{T}$, and each column $\mathrm{v}$ of $\mathrm{V}$ is an eigenvector that corresponds to the nonzero eigenvalue of $\mathbf{B}^{T} \mathbf{B} . \Omega$ is shown according to nonzero eigenvalue of $\mathbf{B B}^{T}$. Each column of $U$ corresponds to a magnetic field pattem the temporal behavior of which is given by the corresponding row of matrix $\mathrm{V}$. The relative strengths of these orthogonal field patterns are given by the diagonal element of $\Omega$. $U$ is divided into noise and signal subspace by singular values, which are affected 


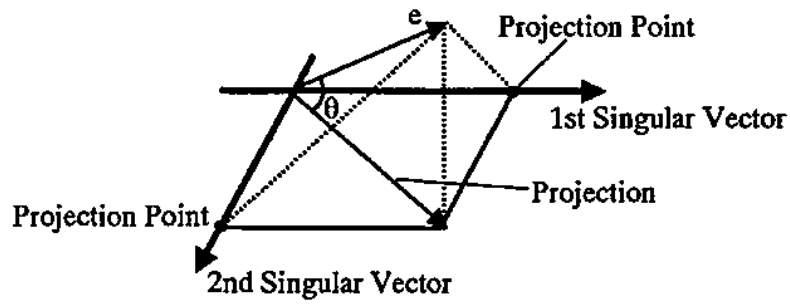

Fig. 2. The projection vector and the projection angle $\theta$.

with the signal to noise ratio (SNR). When SNR is greater than 5 , the signal subspace of $U$ is decided by dominant singular values. The parameters of the sources are so selected that the magnetic field of the sources is included in signal subspace $\mathbf{U}$. In source estimation we employ the following cost function (4):

$$
f=\frac{\mathrm{e}^{T} U U^{T} \mathrm{e}}{\|\mathrm{e}\|\left\|U U^{T} \mathrm{e}\right\|}
$$

where $e$ is the magnetic field of a single dipolar source. It is clear that $f=\cos \theta$, where $\theta$ is the angle between e and the subspace spanned by the column vectors of $U$ as indicated in Fig. 2.

The source estimation is carried out by the following procedure:

1) The magnetic field data is analyzed by singular value decomposition. The number of the sources is lased on the number of dominant singular values.

2) The initial parameters of the sources are determined by tangential magnetic field extremes since tangential magnetic field shows maximum or minimum field just above the location of a source [1].

3) The projection of the magnetic field that single estimating source generates is calculated, and it is evaluated by cost function $\mathrm{f}$.

4) The parameters are searched by the simplex method, and the parameters are adjusted to maximize cost function $\mathcal{f}$.

5) The parameters are determined by the source reaching the convergence criterion.

6) The procedure 3)-5) are repeated for all sources determined in 1) and 2).

This method also uses the singular value decomposition looking like to MUSIC [3]. MUSIC method estimates source localization as matrix independent of noise subspace of $U$. But the proposed method estimates the dipolar source by pattern matching between signal subspace of $U$ and projection of the estimating source. It is difficult to estimate multiple sources using the least squares method, because estimating parameters increase in proportion to the number of sources. But the estimating parameters in the proposed method are constant for using pattern matching by single dipolar source.

\section{COMPUTER SimULATION}

In order to assess this algorithm, inverse calculation to the MEG model consisting of three sources located in the brain was performed. Fig. 3 shows the locations and waveforms of the three sources. The frequency of $S_{1}$ and $S_{2}$ was $20 \mathrm{~Hz}, S_{3}$ was
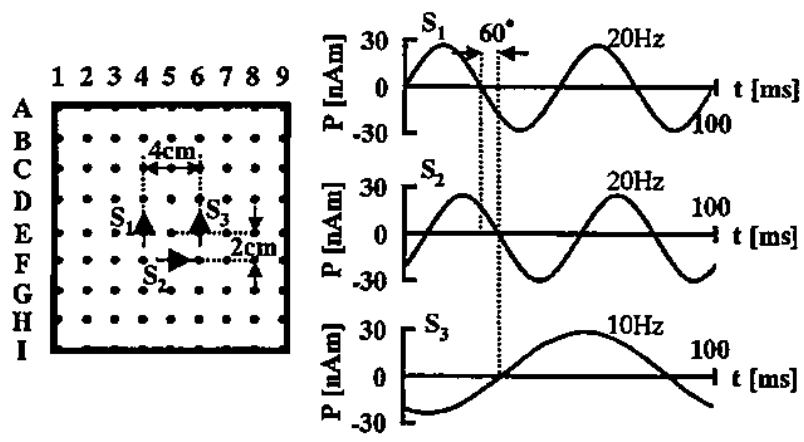

Fig. 3. The locations and waveforms of three sources. Phase difference between $S_{1}$ and $S_{2}$ is the 60 degrees, phase difference of $S_{1}$ and $S_{3}$ is the 60 degrees. The depths of all sources are $2 \mathrm{~cm}$ from the surface of the spherical model.

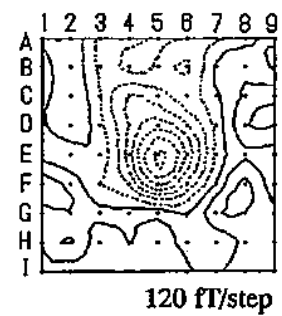

(a) $\mathrm{Br}$ component

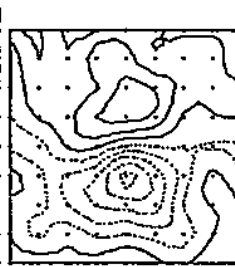

$22 \mathrm{fT} / \mathrm{step}$

(b) $\mathrm{B} \theta$ component

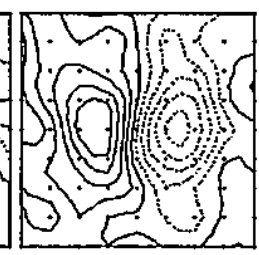

$22 \mathrm{fT} / \mathrm{step}$

(c) $\mathrm{B} \phi$ component
Fig. 4. Isofield contour maps generated by three sources shown in Fig. 3. (time: $5 \mathrm{~ms}$, noise: $20 \%$ ).

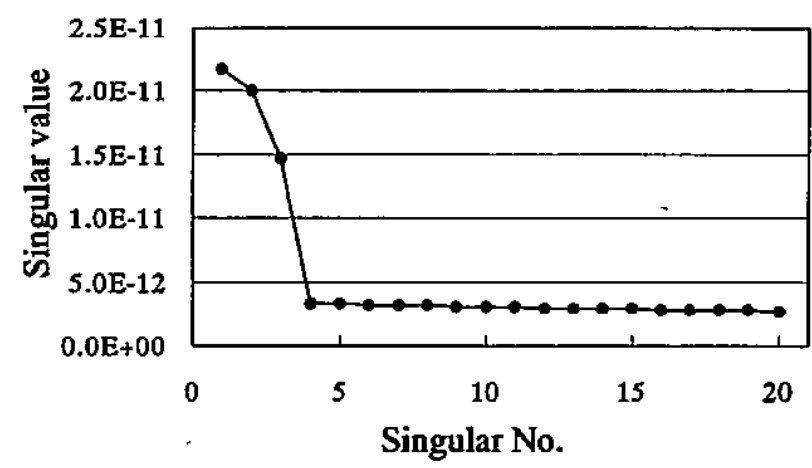

Fig. 5. Result of singular value decomposition of three sources.

$10 \mathrm{~Hz}$. Phase difference of $S_{1}$ and $S_{2}$ was the 60 degrees, phase difference of $S_{1}$ and $S_{3}$ was the 60 degrees. The depths of all sources were $2 \mathrm{~cm}$ from the surface of the spherical model corresponding to human sensory area [9]. A random noise (20\% of maximum value) was applied to the simulation data.

Fig. 4 shows an example of isofield contour maps generated by the three sources shown in Fig. 3 (latency: $5 \mathrm{~ms}$ ). Solid lines show the positive field. As shown in Fig. 4(a), the isofield contour map of $\mathrm{Br}$ shows one extremum and not dipolar. This map was not helpful in estimating the location and the number of sources.

First, we analyzed this data by singular value decomposition shown in Fig. 5. Three dominant singular values were found, so the number of sources was decided as three. Second, the initial location of sources was decided at F5 ( $-119 \mathrm{fT}), \mathrm{E} 6(-114 \mathrm{fT})$ 


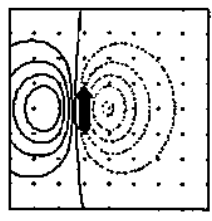

$61 \mathrm{fT} / \mathrm{step}$

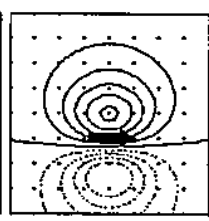

$61 \mathrm{fT} / \mathrm{step}$

(a) $\mathrm{Br}$ component

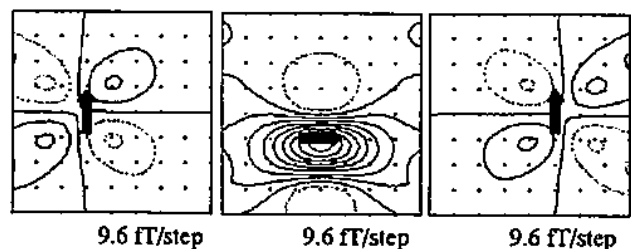

(b) $\mathrm{B} \theta$ component

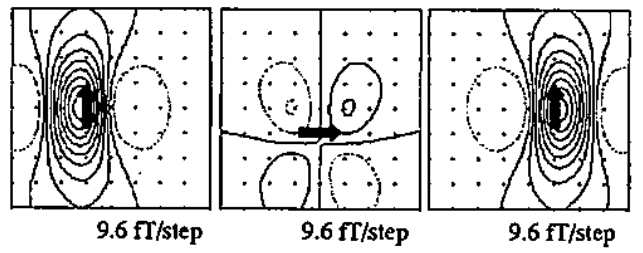

(c) $\mathrm{B} \phi$ component

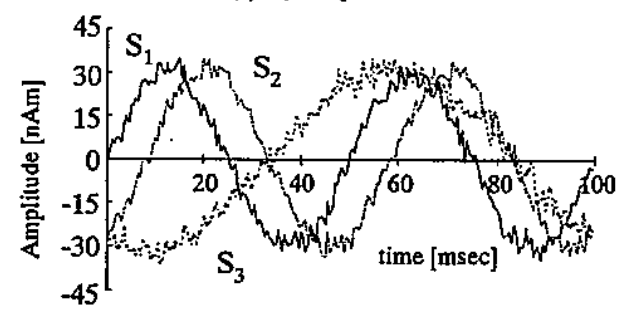

(d) waveforms of three sources

Fig. 6. Isofield counter maps and waveforms generated by the estimated three sources. The arrows show the locations and directions of estimated sources.

and E4 (101 fT) from tangential magnetic field extremes shown in Fig. 4(b), (c). Next, the source estimation was performed using the proposed algorithm.

Fig. 6 shows isofield contour maps and waveforms generated by the estimated three sources. The arrows show the locations and directions of the estimated sources. The estimation errors
$S_{1}, S_{2}$ and $S_{3}$ were $0.2 \mathrm{~mm}, 0.5 \mathrm{~mm}$ and $0.5 \mathrm{~mm}$ respectively. The value of the define evaluation function for all three sources was 0.99 . We were able to estimate three sources over lapping in time using the proposed algorithm.

\section{CONCLUSION}

We propose a new source estimation method using spatiotemporal data of 3-D MEG and singular value decomposition analysis. This method uses tangential component to decide the initial parameters for source analysis. The tangential component provided information about the source location by visual inspection. In order to assess this algorithm, inverse calculation to the MEG model consisting of three sources located in the brain was performed. The results showed that three sources were clearly discriminated, and that the estimation error was less than $1 \mathrm{~mm}$ with SNR $=\overline{5}$. An algorithm proposed here is useful method for estimating multiple sources overlapping in time.

\section{REFERENCES}

[1] K. Kobayashi and Y. Uchikawa, "Estimation of multiple sources using a three-dimensional vector measurement of a magnetoencephalogram," J. Appl. Phys., vol. 83, pp. 6462-6464, 1998.

[2] O. Oshiro, M. Mukai, F. Takeuchi, and S. Kuriki, "Analysis of errors in neuromagnetic localization of multiple current dipole sources," Phys. Med. Biol., vol. 37, pp. 845-852, 1992

[3] J. C. Mosher, P. S. Lewis, and R. M. Leahy, "Multiple dipole modeling and localization from spatio-temporal MEG," IEEE Trans. Biomed. Eng., vol. 39, pp. 541-557, 1992.

[4] Y. Saito, E. Itagaki, and S. Hayano, "A formulation of the inverse problems in magnetostatic fields and its application to a source searching of the human eye fields," J. Appl. Phys., vol. 67, pp. 5830-5832, 1990.

[5] C. Baumgartner, W. W. Sutherling, S. Di, and D. S. Barth, "Spatiotemporal modeling of cerebral evoked magnetic fields to median nerve stimulation," Electroencephalogr. Clin. Neurophysiol., vol. 79, pp. 27-35, 1991.

[6] A. Achim, F. Richer, and J. S. Hilaire, "Methodological considerations for the evaluation of spatio-temporal source models," Electroencephalogr. Clin., vol. 79, pp. 227-240, 1991.

[7] S. Supek and C. J. Aine, "Simulation studies of multiple dipole neuromagnetic source localization: Model order and limits of source resolution," IEEE Trans. Biomed. Eng., vol. 40, pp. 529-540, 1993.

[8] K. Kobayashi and Y. Uchikawa, "Development of a three-dimensional biomagnetic measurement system with 39-channel SQUIDs," in Recent Advances in Biomagnetism, T. Yoshimoto, M. Kotani, S. Kuriki, H. Karibe, and N. Nakasato, Eds, Japan: Tohoku University Press, 1999.

[9] R. Hari, K. Reinikainen, and K. Kaukoranta, "Somatosensory evoked cerebral magnetic fields from SI and SII in man," Electroncephalogr Clin., vol. 57, pp. 254-263, 1984. 